\title{
Long-term event-Holter monitoring in low-populated areas as a valuable diagnostic tool in the detection of arrhythmia
}

\author{
Michał Harańczyk, MD¹, Małgorzata Konieczyńska, MD, PhD¹, Wojciech Płazak, MD, PhD, Prof
}

Harańczyk M, Konieczyńska M, Plazak W. Long-term event-Holter monitoring in low-populated areas as a valuable diagnostic tool in the detection of arrhythmia. Curr Res Cardiol 2017;4(4):69-72.

OBJECTIVES: The purpose of the present study was to implement eventHolter tele-ECG system in small-town area with poor access to healthcare.

METHODS: One hundred patients aged over 55 with suspected arrhythmia were studied for one month, using 4-electrode event-Holter device. The study protocol included 3-times daily acquisition of ECG during complaints. For the period of observation patients were supported by a technical help-desk, psychologist, dietician and physicians.
RESULTS: During this study patients recorded over 7000 ECGs, with pathology rate of $19 \%$. Quality of recordings remained high (88\% ECGs of good quality), and patient's satisfaction was excellent. Clinical impact was reflected in change of treatment in 16 patients, referring to cardiology clinic in 4 patients or cardiac ambulatory treatment in 33 individuals. CONCLUSION: We found event-Holter monitoring as a valuable tool, that may be helpful in daily arrhythmia management. Prolonged time of acquisition may help to register arrhythmia otherwise remaining undetected.

Key Words: Telemedicine; eHealth; Arrhythmias; Telemetry

\section{TABLE 1}

Demographic and clinical characteristics of the study group for right diagnosis and proper treatment to document and recognize the underlying heart rhythm during symptoms. The leading role in diagnostic process of arrhythmias, due to availability and low cost, occupy a classic 12 lead ECG and Holter monitoring (1). Both methods are well known and used from many years, and their clinical usefulness has its justification in everyday clinical practice (2). Due to the rare presence of some arrhythmias, in case of symptoms presented less than daily - these methods may not be sufficient. According to the ESC recommendations of suspected arrhythmias occurring every week to every month, the use of devices such as event-Holter should to be considered. It has potential benefits especially in the context of rare episodes of atrial fibrillation (AF) or atrial flutter (AFl), both increasing the risk of thrombo-embolic complications, as do the sustained forms. External loop recorders play significant role in the diagnosis of arrhythmia, as well as in monitoring of the treatment (3). In case of reported complaints on palpitations, event-Holter allows temporal correlation of reported problems with the current ECG (4).

This study was carried out as a part of ADAPT2DC EU project, with main goals as achieving better access to health care in depopulating areas and providing high quality medical service for elderly patients. The aim of this pilot study was to create a model of ECG telemonitoring (tele-ECG) and remote care of patients over the age of 55 years, living in areas of small towns with a reduced-access to specialized cardiac care

\section{METHODS}

One hundred patients aged over 55 years with suspected arrhythmias, history of presyncope or syncope were enrolled for this study. Patients were selected from 4 districts in the southern Poland, with the average number of the population between 50,000 and 100,000 citizens. Patients were recruited and examined in the period from March to October 2013. In the closest relatives of 19 patients sudden cardiac death was suspected as a cause of death. In the study group 78 patients presented any kind of arrhythmia at baseline, 33 reported faints in past, 71 reported presyncope. 13 patients had paroxysmal atrial fibrillation (PAF), and 2 persistent atrial fibrillations. 9 patients have undergone in-hospital antiarrhythmic therapy with antiarrhythmic agents or electrical cardioversion (AF or supraventricular tachycardia-SVT). Three patients have undergone cardiac arrest ( 2 during in-hospital surgical procedures and 1 after choking), 2 had a pacemaker (due to tachy-brady syndrome and sick-sinus syndrome), what was allowed by inclusion criteria (Tables 1 and 2).

\begin{tabular}{ccc}
\hline Variables & Women & Men \\
\hline N.(\%) & $66(66 \%)$ & $34(34 \%)$ \\
\hline Mean age (years) & $65.3 \pm 6.0$ & $66.1 \pm 7.2$ \\
Project attendance (days) & $29 \pm 4$ & $29 \pm 2$ \\
ECGs recorded per patient (N.) & $70 \pm 28$ & $72 \pm 28$ \\
Mean SBP (mmHg) & $136 \pm 23$ & $138 \pm 17$ \\
Mean DBP (mmHg) & $81 \pm 13$ & $85 \pm 12$ \\
Arterial hypertension & $48(48 \%)$ & $30(30 \%)$ \\
Heart failure & $12(12 \%)$ & $3(3 \%)$ \\
Ischemic heart disease & $26(26 \%)$ & $1313 \%)$ \\
Past myocardial infarction & $6(6 \%)$ & $5(5 \%)$ \\
Past cardiosurgery procedure & $2(2 \%)$ & $2(2 \%)$ \\
Pulmonary disease* & $8(8 \%)$ & $4(4 \%)$ \\
\hline
\end{tabular}

*Pulmonary diseases: Asthma (5 patients), COPD, Sarcoidosis

TABLE 2

Drug characteristics

\begin{tabular}{cc}
\hline Drugs & \% of patients \\
\hline Betablockers & 59 \\
ACEi/ARB & 56 \\
Diuretics & 45 \\
CCB & 22 \\
Antiarrhythmics & 7 \\
Hypoglycemic agents & 12 \\
Lipid lowering agents & 40 \\
ASA & 47 \\
Anticoagulants & 12 \\
Anticholinergics & 1 \\
Ivabradine & 1 \\
Nitrats & 1 \\
Magnesium supplements & 20 \\
Potassium supplements & 21
\end{tabular}

ACEi: Angiotensin-Converting-Enzyme Inhibitors; ARB: Angiotensin Receptor Blockers; ASA: Acetylsalicylic Acid; CCB: Calcium Channel Blockers

${ }^{1}$ Department of Diagnostic Medicine, John Paul II Hospital in Kraków, Poland; ${ }^{2}$ Department of Cardiac and Vascular Diseases, John Paul II Hospital, Institute of Cardiology, Jagiellonian University, Poland

Correspondence: Dr. Michał Harańczyk, MD, Department of Diagnostic Medicine, John Paul II Hospital in Kraków, Poland. Telephone +48 533663326 , fax +48 126142556, email michal.haranczyk@gmail.com

Received: October 30, 2017, Accepted: December 01, 2017, Published: December 04, 2017

This open-access article is distributed under the terms of the Creative Commons Attribution Non-Commercial License (CC BY-NC) (http://
OPEN
creativecommons.org/licenses/by-nc/4.0/), which permits reuse, distribution and reproduction of the article, provided that the original work is
properly cited and the reuse is restricted to noncommercial purposes. For commercial reuse, contact reprints@pulsus.com 
Out of 100 patients, 17 were referred by cardiologist, 31 by general practitioners (GPs) and 21 by specialist of internal medicine. All participating patients gave written informed consent. All investigations were in accordance with the Declaration of Helsinki. ECG recordings were performed using a 4-electrode device (EHO-MINI EVENT HOLTER, Pro-Plus Sp.z o.o.), allowing recordings of the 7-lead ECG (6 standard limb leads and one precordial lead V2) in the on-demand setting, and bearing CE Mark. Each event-Holter device had an embedded SIM card from a mobile network operator with sufficient mobile phone coverage in the area where the patient lived. ECG-trace consisted of the ECG for 30 seconds before pressing the button (looping buffer), and 30 seconds after-a total of 1-minute recording of each trace was stored at once. Patients were trained by technicians how to use the device, place the electrodes correctly, and send the ECG to the Telemedicine Center in the Department of Diagnostic Medicine. In case of symptoms (fainting, loss of consciousness, dizziness, other disturbing), the patient had to press the button for approx. 6 seconds, while avoiding sudden movements. The signal was transferred automatically via cellular network (built-in GSM/GPRS). If on a particular day there were no symptoms, patients were asked to send 3 records at different times during the day. In addition, patients were asked to keep a diary with submitted entries and selected incidents. The stored data were transmitted to the healthcare provider for analysis. ECG traces were collected on a data server located in the hospital. In addition, researchers had a round-the-clock access to the digitally stored ECG and to the patients' electronic health records (EHR) via VPN connection (virtual private network). Every recording was analyzed by a cardiologist. In case of diagnostic uncertainty, a senior cardiologist was available for case conference. There was an available cardiologist on call in 24/7 setting, in case of patients quotes. During office hours a help-desk service was available for the patients as well. One-month recording period was preceded by "start day" taking place in hospital, consisting of various consultations (cardiologic, dietitian, psychological, and device-orientated technical training). At the end of follow-up, a control clinical and dietitian visit has taken place. Moreover, during the study every patient underwent laboratory tests (fasting glucose level, cholesterol level).

\section{RESULTS}

Out of 100 consecutive patients enrolled in the study, 3 patients withdrew from participation during the course of the study. All 3 as a reason for the resignation reported technical difficulties with the managing the device. A total of 7003 ECG traces were recorded, and $88 \%$ was of good quality. Each patient recorded daily an average of 2.4 traces $(2.4 \pm 0.91)$. The average contribution time of the patients in this study was 30 days $(29.7 \pm 1.9)$. The registered pathological traces have accounted for $19 \%$ of all recordings. Among all pathological traces, 21 (2\%) were of bad quality of the recordings what didn't allow for a full interpretation of the arrhythmia (Figure 1).

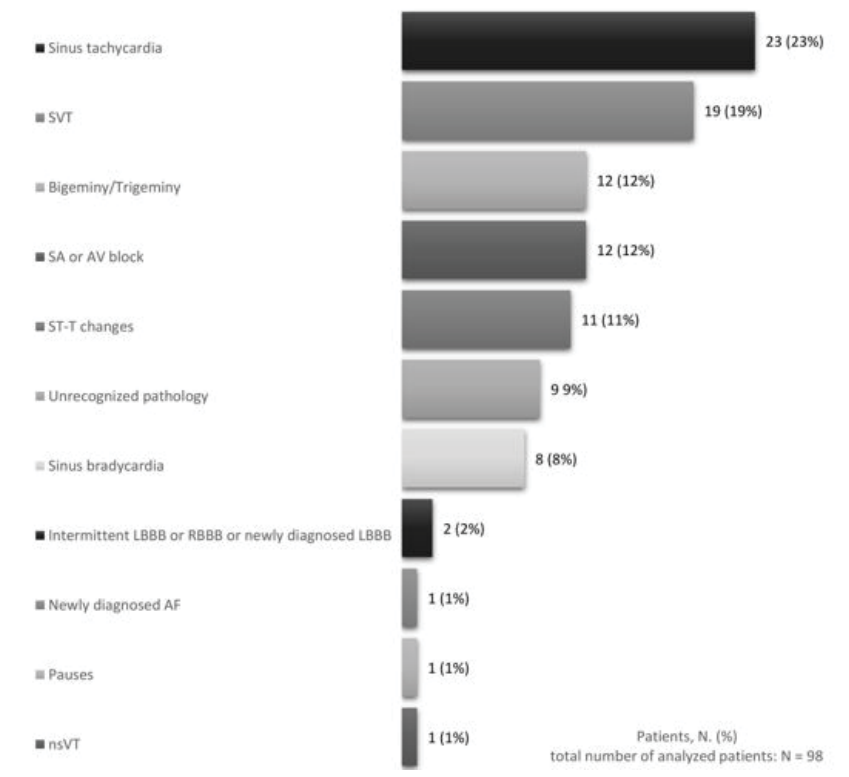

Figure 1) Characteristics of arrhythmias

SVT: Supraventricular Tachycardia; SA or AV block: Sinoatrial or Atrioventricular Block; LBBB: Left Bundle Branch Block; AF: Atrial Fibrillation; nsVT: Non-Sustained Ventricular Tachycardia; ST-T Changes: Abnormalities in ST Segment or T Wave Related to Ischemia

\section{Paroxysmal atrial fibrillation}

In our study PAF occurred in 13 patients at baseline, in 2 patients as a sustained or persisted AF. During the study we diagnosed one new case of PAF. In 4 patients without a history of AF, episodes of irregular paroxysmal tachycardia with narrow QRS were recorded, but poor quality of recordings did not allow to form a reliable diagnosis.

All 4 patients reported a history of palpitations / irregular heart rate; in 2 of them few episodes of regular SVT were recorded as well.

\section{Ventricular arrhythmias}

Ventricular arrhythmias (VA) were reported in 11 patients at baseline. During the study VA were present in $42 \%$ of patients. No sustained forms of VA have been registered, in one patient there was one non-sustained ventricular tachycardia (nsVT) consisted of 6 beats (Table 3).

\section{TABLE 3}

\section{Lown's grading system for ventricular arrhythmias}

\begin{tabular}{cc}
\hline LOWN Grade & $\%$ of patients \\
\hline 0 & 58 \\
1 & 14 \\
2 & 11 \\
$3 \mathrm{~A}$ & 0 \\
$3 \mathrm{~B}$ & 12 \\
$4 \mathrm{~A}$ & 4 \\
$4 \mathrm{~B}$ & 1 \\
5 & 0 \\
\hline
\end{tabular}

Bradyarrhythmia and conduction blocks

Bradycardia, pauses and sinoatrial or atrioventricular blocks were present in 15 patients. Feeling of slow heart rhythm with or without concomitant feeling of arrhythmia was present in 10 patients at baseline (both patients with cardiac pacemaker were present in this group). In this subgroup, bradycardia $<40 / \mathrm{min}$. was present only in one patient during the observation, there were SVT present in 3 patients; pauses $>2$ sec. was present only in 1 patient and in 2 patients there were no abnormalities in recorded traces.

\section{Tachyarrhythmia}

Palpitations and feeling of tachyarrhythmia were reported in 47 patients at baseline-in this subgroup in 10 patients there was no pathology during the study. During the observation tachyarrhythmia was present in 40 patients. In 9 patients poor quality of some recordings has been registered, where a pathology was highly suspected. We were unable to set up a final diagnosis, nevertheless, on the basis of ECG-morphology, in 6 patients a suspicion on SVT was aroused (unrecognized tachyarrhythmia). A total of 11 traces has been recorded, with suspicion on SVT, what counts for $6 \%$ of all recorded tachyarrhythmias.

\section{Quality of recordings}

We achieved a high level of good quality ECG traces $-88 \%$ of all recorded traces were classified as good or moderate quality, and analyzed by a cardiologist. In the case of records with poor quality, they were not interpreted unless any of the pathologies were clearly seen in the recordings.

\section{Patient satisfaction}

In a survey conducted at the end of the project, the following questions: "whether the service had a positive impact on improving the quality of life", "did this service improved your sense of security", and "whether you would like to use this service in the future","yes" or "definitely yes," answered respectively- $83 \%, 94 \%$ and $92 \%$ of respondents, what corresponds with the positive perception of mobile services by patients in other studies (5). Only 3 patients withdrew from further participation in the project because of technical problems with using the device. The most common technical problem during the study was poor quality of transmitted records, probably resulting from physical activity and improper electrode contact with the surface of the body. Episodes of confusion between the sides of the electrodes placement described in the literature (5), took place only in a few records in one patient.

\section{Correlation of ECG-findings with symptoms}

At baseline symptoms were classified into 3 group: presyncope, syncope and arrhythmia, according to complaints. Thus, 71 patients have reported 
presyncope in past, 33 syncope, 78 arrhythmias, and 19 patients have complained of all 3 types of symptoms. The highest number of patients with pathologic traces $(82 \%)$ were registered in group with arrhythmia at baseline; in comparison to patients with presyncope at baseline (73\%), syncope $(63 \%)$ and all 3 symptoms presented at baseline (58\%), respectively.

\section{Impact of the results on further treatment}

After completing the study, the patients were consulted as to change in treatment, further diagnostics in an appropriate ambulatory, or planned admission to the hospital (there was no need for acute admissions in this study). Changes in treatment caused directly by the result of Holter monitoring concerned starting of anticoagulation in 2 patients. Moreover, there was a change in hypertension treatment (6 patients), dyslipidemia treatment ( 8 patients) and hyperglycemia treatment ( 3 patients). In 5 individuals reported to electrophysiology laboratory a relevant bradycardia was confirmed-in 2 patients episodes of sinus bradycardia with fragments of escape-rhythm (ventricular and supraventricular origin), in 1 patient an sinoatrial block, in 1 patient an intermittent LBBB. In 2 patients a number of ventricular arrhythmias were diagnosed. The reason for the further evaluation in cardiology clinic were: ischemic changes in 3 patients, relevant bradycardia in 1 patient (Figure 2).

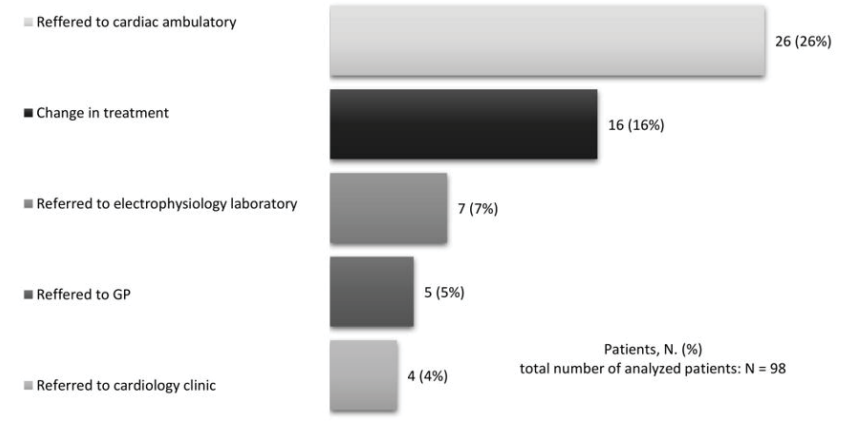

Figure 2) Patient management

Ambulatory consultation with cardiologist was advised in 4 patients because of bradycardia, in 6 patients due to sinus tachycardia, in 4 patients with ventricular bigeminy/trigeminy, in 7 patients with supraventricular tachycardia, and in 4 patients with atrioventricular block (Mobitz I in 3 patients and Mobitz II in 1).

\section{DISCUSSION}

The main finding of the present study consists in a confirmation of eventHolter devices as an important and valuable tool in detecting cardiac arrhythmias. Implementation of event-Holter in the present study has changed the treatment or has initiated more advanced diagnostic process in $45 \%$ of patients.

ECG telemonitoring is a recognized diagnostic tool for arrhythmia detection, which has been proven in numerous clinical trials. There are numerous data concerning the elderly population, but the majority of these studies refer to the use of ECG for pre-hospital assessment in patients with suspected myocardial ischemia or acute coronary syndrome (6-8). There are only few trials concerning wide spectrum of ECG changes in elderly patients living in low-populated areas $(9,10)$. This work is one of the few publications that refers to the patients over 55 years old, living areas covered by long-term ECG telemonitoring based on event-Holter device.

Proper diagnosis of cardiac arrhythmias may constitute a great challenge, especially in case of paroxysmal arrhythmias and a relatively rare occurrence. In the areas with reduced access to high quality medical care, it may be helpful to use remote detection of arrhythmias, e.g. event-Holter (11). These devices monitor heart rhythm over a longer period than Holter monitoring, and the patients activate recording only during periods of discomfort by pressing the appropriate button. Application of solutions for remote data transmission (GSM, GPRS, EDGE, $3 \mathrm{G}$ etc.) allows instant transfer of data to a remotely located clinical center. Acute care centers working in 24-hour mode are able to continually analyze reported symptoms, obtained recordings, and to provide adequate support to the patients (12). Disadvantages of eventHolter include the need to constantly maintain connection of the device to the chest via electrodes, which may result in local irritation of the skin and adversely affect the compliance of the patients (13). In the case of loss of consciousness, when the time of regaining consciousness exceeds the programmed loop-time, it is highly unlikely to record arrhythmia $(14,15)$. In rare cases such devices can be activated e.g. by a trained family member. Another disadvantage we learned from this study is the time-consumptive patients' training, in order to learn basic device management, as well as a need to maintain long-time technical support as a patients' back-up.

Event monitoring devices are handy and convenient, as opposed to Holter ECG devices limiting patients daily activity to small extent. This model of monitoring also meets with good acceptance of patients. Due to high sensitivity and the extended time of registration, it becomes possible to record rare occurred arrhythmias. Our study has shown an increased arrhythmia diagnostic yield by use of prolonged event monitoring, with high arrhythmia number being recognized. Even though some studies have demonstrated high efficacy of 7-14 days observation (16), our prolonged protocol didn't affect the compliance in study, what reminded very high. In fact, the service was appreciated as the one what gives a feeling of safety and confidence. The achieved quality of the recordings is comparative to a study of Alte et al. who reported $94 \%$ good ECGs (17), but significantly better than in the study Singh et al. (11), who reported $80 \%$ of good quality ECGs. In another study (5) the authors reached $97 \%$ of good quality traces, but the length of each trace was significantly shorter (10 sec. vs $30 \mathrm{sec}$. in our study). Successful transmission-rate was $100 \%$, which corresponds to the current results of leading research (18).

\section{CONCLUSION}

The most common pathologies found in study were sinus tachycardia and SVT. They were also the most common finding in all subgroups related to symptoms complained at baseline. Detection of tachyarrhythmias enabled change in treatment in $9 \%$ of patients, and implementation of further cardiac diagnostic in 19\% individuals. The level of recognized AF in our study remained significantly lower than in comparable studies (19), due to different criteria for recruiting patients allowing attendance to individuals with known arrhythmia. In elderly patients $\mathrm{AF}$ is associated with a nearly $25 \%$ rise in the risk of stroke (20). Moreover, in next 50-years a twofold increase in number of adults 55 years and over with AF in the European Union is estimated (21). AF is present in $3.7 \%$ to $4.2 \%$ of those aged $60-70$ years, and in $10 \%$ to $17 \%$ of those aged 80 years or older. Additionally, it occurs more commonly in males (male/female ratio 1.2:1) (22). In our study, $\mathrm{AF}$ was present in 16 patients, in next 4 patients high probability of this arrhythmia was stated, which corresponds to an average incidence of $\mathrm{AF}$ in the general population. In this subpopulation syncope was reported in $40 \%$ patients, presyncope in $75 \%$ and all patients reported feeling of arrhythmia.

A large group of registered arrhythmias were premature atrial and ventricular contractions (PACs and PVCs), founded in 6\% and $11 \%$ patients, respectively, what corresponds with other studies $(4,22)$.

The use of event-Holter with multiple lead allowed also the diagnosis of ischemic changes in 7 patients, 2 of which were subsequently referred for hospitalization and 5 for further outpatient diagnostics. Diagnosis of ischemic changes, like ST-T abnormalities, is impossible in single-lead devices. Therefore, implementation of multi-lead event-Holter may increase detection rate of transient ischemia or serious incidents, like myocardial infarction (23). Other possible advantages of multi-lead devices, like a detection of arrhythmia with the shift of electrical axis or aberration in QRS complexes was not confirmed in our study.

Therefore, we postulate, that event-Holter monitoring may be an effective tool in diagnostics of arrhythmias and have a significant impact on further patient management. Extended period of heart rhythm recording may help to register arrhythmia otherwise remaining undetected. As an efficient and convenient method with high diagnostic sensitivity in the field of arrhythmia detection, it's further deployment at a large scale into clinical practice should be expected.

\section{ACKNOWLEDGEMENT}

This study was granted by Marshal of the Malopolska Region as a part of ADAPT2DC - Adaptation to demographic change project

\section{DISCLOSURE}

The authors have no conflicts of interest to declare.

\section{REFERENCES}

1. Paudel B, Paudel K. The diagnostic significance of the Holter monitoring in the evaluation of palpitation. J Clin Diagn Res. 2013;7(3):480. 
2. Brignole M, Auricchio A, Baron-Esquivias G, et al. 2013 ESC guidelines on cardiac pacing and cardiac resynchronization therapy: The task force on cardiac pacing and resynchronization therapy of the European Society of Cardiology (ESC). Developed in collaboration with the European Heart Rhythm Association (EHRA). Europace. 2013;15(8):1070-18.

3. Fuster V, Ryden LE, Cannom DS, et al. ACC/AHA/ESC 2006 guidelines for the management of patients with atrial fibrillation executive summary: A report of the American College of Cardiology/ American Heart Association Task Force on practice guidelines and the European Society of Cardiology Committee for Practice Guidelines (Writing Committee to Revise the 2001 Guidelines for the Management of Patients with Atrial Fibrillation) Developed in collaboration with the European Heart Rhythm Association and the Heart Rhythm Society. Eur Heart J. 2006;27(16):1979-2030.

4. Kirtava Z, Gegenava T, Gegenava M, et al. Mobile telemonitoring for arrhythmias in outpatients in the republic of georgia: A brief Report of a pilot study. Telemed E-Health. 2012;18(7):570-1.

5. Rubel P, Fayn J, Nollo G, et al. Toward personal eHealth in cardiology. Results from the EPI-MEDICS telemedicine project. J Electrocardiol. 2005;38(4):100-6.

6. BrunettiND, Di Pietro G, Aquilino A, etal. Pre-hospital electrocardiogram triage with tele-cardiology support is associated with shorter timeto-balloon and higher rates of timely reperfusion even in rural areas: data from the Bari-Barletta/Andria/Trani public emergency medical service 118 registry on primary angioplasty in ST-elevation myocardial infarction. Eur Heart J Acute Cardiovasc Care. 2014;3(3):204-13.

7. Papai G, Racz I, Czuriga D, et al. Transtelephonic electrocardiography in the management of patients with acute coronary syndrome. J Electrocardiol. 2014;47(3):294-9.

8. Brunetti ND, Gennaro LD, Amodio G, et al. Telecardiology improves quality of diagnosis and reduces delay to treatment in elderly patients with acute myocardial infarction and atypical presentation. Eur J Cardiovasc Prev Rehabil. 2010;17(6):615-20.

9. Fetsch T. Prevention of atrial fibrillation after cardioversion: Results of the PAFAC trial. Eur Heart J. 2004;25(16):1385-94.

10. Scalvini S, Piepoli M, Zanelli E, et al. Incidence of atrial fibrillation in an Italian population followed by their GPs through a telecardiology service. Int J Cardiol. 2005;98(2):215-20.

11. Singh M, Agarwal A, Sinha V, et al. Application of handheld tele-ECG for health care delivery in Rural India. Int J Telemed Appl. 2014;2014:1-6.
12. Leshem-Rubinow E, Berger M, Shacham J, et al. New real-time Loop recorder diagnosis of symptomatic arrhythmia via telemedicine. Clin Cardiol. 2011;34(7):420-5.

13. Schuchert A. Event-Rekorder. Herzschrittmachertherapie Elektrophysiologie. 2008;19(3):130-6.

14. Zimetbaum P, Kim KY, Ho KK, et al. Utility of patient-activated cardiac event recorders in general clinical practice. Am J Cardiol. 1997;79(3):371-2.

15. Roche F, Gaspoz JM, Pichot V, et al. Accuracy of an automatic and patient-triggered long-term solid memory ambulatory cardiac event recorder. Am J Cardiol. 1997;80(8):1095-8.

16. Barrett PM, Komatireddy R, Haaser S, et al. Comparison of 24-hour holter monitoring with 14-day novel adhesive patch electrocardiographic monitoring. Am J Med. 2014;127(1):95.e11-95.e17.

17. Alte D, Völzke H, Robinson DM, et al. Tele-electrocardiography in the epidemiological "Study of Health in Pomerania" (SHIP). J Telemed Telecare. 2006;12(2):103-7.

18. Dhruva VN, Abdelhadi SI, Anis A, et al. ST-segment analysis using wireless technology in acute myocardial infarction (STAT-MI) trial. J Am Coll Cardiol. 2007;50(6):509-13.

19. Hendrikx T, Rosenqvist M, Wester P, et al. Intermittent short ECG recording is more effective than 24-hour Holter ECG in detection of arrhythmias. BMC Cardiovasc Disord. 2014;14(1):1.

20. Annoni G, Mazzola P. Real-world characteristics of hospitalized frail elderly patients with atrial fibrillation: Can we improve the current prescription of anticoagulants? J Geriatr Cardiol. 2016;13(3):226-32.

21. Krijthe BP, Kunst A, Benjamin EJ, et al. Projections on the number of individuals with atrial fibrillation in the European Union, from 2000 to 2060. Eur Heart J. 2013;34(35):2746-51.

22. Scherr D, Dalal D, Henrikson CA, et al. Prospective comparison of the diagnostic utility of a standard event monitor versus a "leadless" portable ECG monitor in the evaluation of patients with palpitations. J Interv Card Electrophysiol. 2008;22(1):39-44.

23. Nikus K, Lähteenmäki J, Lehto $P$, et al. The role of continuous monitoring in a $24 / 7$ telecardiology consultation service-A feasibility study. J Electrocardiol. 2009;42(6):473-80. 